\title{
THE THEORY OF ORDERED ABELIAN GROUPS DOES NOT HAVE THE INDEPENDENCE PROPERTY
}

\author{
BY
}

Y. GUREVICH ${ }^{1}$ AND P. H. SCHMITT ${ }^{2}$

\begin{abstract}
We prove that no complete theory of ordered abelian groups has the independence property, thus answering a question by B. Poizat. The main tool is a result contained in the doctoral dissertation of Yuri Gurevich and also in P. H. Schmitt's Elementary properties of ordered abelian groups, which basically transforms statements on ordered abelian groups into statements on coloured chains. We also prove that every $n$-type in the theory of coloured chains has at most $2^{n}$ coheirs, thereby strengthening a result by $\mathrm{B}$. Poizat.
\end{abstract}

0. Background and introduction. The independence property is one of the properties introduced by S. Shelah in $[\mathbf{1 6}]$.

DEFINITION. A complete theory $T$ is said to have the independence property, if there is a formula $\varphi(\vec{x}, \vec{y})$ such that for every $k>0$ :

$$
T \cup\left\{\exists \vec{x}^{s}\left(\bigwedge_{l \in s} \varphi\left(\vec{x}^{s}, \vec{c}^{l}\right) \& \bigwedge_{l \notin s} \neg \varphi\left(\vec{x}^{s}, \vec{c}^{l}\right)\right): s \subseteq k\right\}
$$

is consistent.

To convey an idea of the relevance of this definition we remember two results from (logical) stability theory.

For a structure $\mathfrak{A}$ let $S_{m}(\mathfrak{A})$ denote the set of complete $m$-types over $\mathfrak{A}$. We write $S(\mathfrak{A})$ for $S_{1}(\mathfrak{A})$ and in the following $\mu$ always denotes an infinite cardinal. The stability function $g_{T}$ of a complete theory $T$ is defined by

$$
g_{T}(\mu)=\sup \{\operatorname{card}(S(\mathfrak{A})): \mathfrak{A} \models T \text { and } \operatorname{card}(\mathfrak{A})=\mu\} .
$$

It was proved in [8] building heavily on [16] (see also [9]) that for any countable complete theory $T g_{T}$ is one of the six functions:

$$
\mu, \mu+2^{\omega}, \mu^{\omega}, \operatorname{ded}(\mu), \operatorname{ded}(\mu)^{\omega}, 2^{\mu}
$$

where $\operatorname{ded}(\mu)=\sup \{\lambda$ : there is a linear order $Y$ with $\operatorname{card}(Y)=\mu$ and $Y$ has $\lambda$ Dedekind cuts $\}$. It was already contained in $[\mathbf{1 6}]$ that the independence property basically characterizes theories $T$ with $g_{T}(\mu)=2^{\mu}$. More precisely:

0.1 . If $T$ has the independence property then for all $\mu: g_{T}(\mu)=2^{\mu}$.

0.2. If for some $\mu \operatorname{ded}(\mu)^{\omega}<g_{T}(\mu)$, then $T$ has the independence property.

\footnotetext{
Received by the editors September 7, 1982 and, in revised form, July 25, 1983.

1980 Mathematics Subject Classification. Primary 03C60; Secondary 06F20.

${ }^{1}$ Fellow of the Institute for Advanced Studies of the Hebrew University in Jerusalem during the preparation of this paper.

${ }^{2}$ I gratefully acknowledge financial support by the Deutsche Forschungsgemeinschaft during the preparation of this paper. 
If the generalized continuum hypothesis is true 0.2 does not say anything, since then we have for all $\mu: g_{T}(\mu) \leq 2^{\mu}=\operatorname{ded}(\mu)^{\omega}$. To describe the second situation where the independence property shows up we need the concept of coheir.

Definition. Let $\mathfrak{A}, \mathfrak{B}$ be structures, $\mathfrak{A} \preceq \mathfrak{B}, p \in S_{m}(\mathfrak{A}), q \in S_{m}(\mathfrak{B}) . q$ is called a coheir of $p$ if for every formula $\varphi(\vec{x}, \vec{b}) \in q$ there is $\vec{a} \in \mathfrak{A}$ such that $\mathfrak{B} \models \varphi(\vec{a}, \vec{b})$ and $p \subseteq q$.

B. Poizat characterized in $[\mathbf{1 2}]$ complete theories $T$ with the independence property in terms of the maximal number of coheirs:

0.3 . If $T$ does not have the independence property then for all $\mu \geq \operatorname{card}(T)$ and all models $\mathfrak{A}, \mathfrak{B}$ of $T$ with $\operatorname{card}(\mathfrak{A})=\mu$ and $\mathfrak{A} \preceq \mathfrak{B}$, every $p \in S(\mathfrak{A})$ has at most $2^{\mu}$ coheirs in $S(\mathfrak{B})$.

0.4 . If $T$ has the independence property then there are models $\mathfrak{A}, \mathfrak{B}$ of $T$ with $\mathfrak{A} \preceq \mathfrak{B}, \operatorname{card}(\mathfrak{A})=\mu \geq \operatorname{card}(T)$ such that some $p \in S(\mathfrak{A})$ has $2^{2^{\mu}}$ coheirs in $S(\mathfrak{B})$.

Bruno Poizat has shown in [12] that no complete theory of coloured chains (i.e. linear orders with additional unary predicates) has the independence property. $M$. Parigot proved the same for complete theories of trees $[\mathbf{1 1}]$. In fact they both showed that for any two structures $\mathfrak{A} \preceq \mathfrak{B}$ in the considered theories any type $p \in S(\mathfrak{A})$ has at most $2^{\omega}$ many coheirs in $S(\mathfrak{B})$ regardless of the cardinality of $\mathfrak{A}$. It was for a short while an open question whether this was true for any theory without the independence property. But Françoise Delon [1] constructed a theory without the independence property such that for every $\mu$ there are models $\mathfrak{A}, \mathfrak{B}, \mathfrak{A} \preceq$ $\mathfrak{B}, \operatorname{card}(\mathfrak{A})=\mu$ such that some type $p \in S(\mathfrak{A})$ has at least $\operatorname{ded}(\mu)$ coheirs in $S(\mathfrak{B})$. Examples of theories with the independence property are the complete theories of infinite Boolean algebras. J.-L. Duret proved in $[\mathbf{3}]$ that for every infinite model $K$ of the theory of finite fields, $\operatorname{Th}(K)$ does have the independence property. $\mathrm{F}$. Delon showed in [2] that under the hypothesis of the theorem of Ax and Kochen the theory of a valued field has the independence property only if the theory of its value group or the theory of its residue field has the independence property. It was this result that motivated B. Poizat to ask in $[\mathbf{1 2}]$ whether any complete theory of ordered abelian groups can have the independence property.

In $\S 1$ we strengthen the result of $\mathrm{B}$. Poizat and show that any $n$-type $p$ in a coloured chain has at most $2^{n}$ coheirs. $\S 2$ collects the definitions and theorems from [15] that will be used to prove our main theorem in $\S 3$.

1. Number of coheirs in coloured chains. By a coloured chain we understand a linear order with additional unary predicates. The number of additional unary predicates may be arbitrary unless specified otherwise.

Let $M$ be a coloured chain and $p\left(x_{1}, \ldots, x_{n}\right)$ a type in $S_{n}(M)$. The cut determined by $p$ and $x_{i}$ is defined to be the pair:

$$
A_{i}=\left\{a \in M: a<x_{i} \in p\right\}, \quad B_{i}=\left\{a \in M: x_{i}<a \in p\right\} .
$$

FACT A. If $M$ contains $\kappa$ many additional unary predicates and $\left(A_{1}, B_{1}\right), \ldots$, $\left(A_{n}, B_{n}\right)$ are cuts of $M$ then there are at most $2^{\kappa_{0}}$ many types in $S_{n}(M)$ such that for all $i, 1 \leq i \leq n, p$ and $x_{i}$ determine the cut $\left(A_{i}, B_{i}\right)$, where $\kappa_{0}=\max (\kappa, \omega)$.

This was proved for $n=1$ and $\kappa \leq \omega$ in the course of the proof of Théorème 9 in [12]. There is no new argument needed to verify our slightly more general version.

DEFINITION. If $a, b$ are elements of a coloured chain $A$, we say that a sequence $\varphi_{1}, \ldots, \varphi_{k}$ of formulas is realized between $a$ and $b$ if there are elements $c_{1}, \ldots, c_{k}$ 
of $A$ with $a<c_{1}<\cdots<c_{k}<b$ where each $c_{i}$ satisfies the corresponding formula $\varphi_{i}$.

THEOREM 1.1. Two increasing sequences $a_{1}<\cdots<a_{n}$ from the coloured chain $C$ and $b_{1}<\cdots<b_{n}$ from the coloured chain $D$ have the same type (over the empty set of parameters) provided that:

(i) for each $i, 1 \leq i \leq n, a_{i}$ and $b_{i}$ have the same type,

(ii) for each $i, 1 \leq i \leq n$, the same finite sequences of formulas are realized between $a_{i}$ and $a_{i+1}$ and between $b_{i}$ and $b_{i+1}$.

Theorem 1.1 is stated in M. Rubin's paper [13, p. 408] as Theorem 3.10 without proof. But having read $\S 3$ of $[\mathbf{1 3}]$ it is not hard to supply one. A proof of this theorem is also contained in [14].

THEOREM 1.2. Let $A, C$ be coloured chains, $A \subseteq C$ and $p\left(x_{1}, \ldots, x_{n}\right)$ an n-type over $A$. Then $p$ has at most $2^{n}$ coheirs in $S_{n}(C)$.

Proof. Let $\left(A_{i}, B_{i}\right), 1 \leq i \leq n$, be the cuts of $p$ at $x_{i}$. The subset $C_{i}=\{c \in$ $\left.C: A_{i}<c<B_{i}\right\}$ is called the filling of the cut $\left(A_{i}, B_{i}\right)$ in $C$.

For a coheir $q \in S(C)$ of $p$ there are for each $i, 1 \leq i \leq n$, only the two possibilities that

$$
\left(x_{i}<c\right) \in q \text { for all } c \in C_{i}
$$

in which case we call $q$ a left extension of $p$ at $x_{i}$ or

$$
\left(c<x_{i}\right) \in q \text { for all } c \in C_{i}
$$

and we call $q$ a right extension of $p$ at $x_{i}$.

We will show that two coheirs $q_{1}, q_{2}$ of $p$ with the same distribution of left/right extensions at $x_{1}, \ldots, x_{n}$ already coincide. By Theorem 1.1 it suffices to prove for each $i, 1 \leq i \leq n$, and each $c \in C$ :

(1) $q_{1}$ and $q_{2}$ imply the same finite sequences of formulas be realized between $c$ and $x_{i}$ and the same for sequences between $c$ and $x_{i}$.

Let us assume that $q_{1}$ and $q_{2}$ are both left extensions at $x_{i}$, the case of both being right extensions is completely symmetric. When dealing with the case $c \leq x_{i}$ we may assume without loss of generality that $c \in A$, since we can always find $a \in A_{i}$ such that $c \leq a$. But now (1) follows since the restrictions of $q_{1}$ and $q_{2}$ to types over $A$ coincide. In the case $x_{i} \leq c$ we may assume by the same token that $c \in C_{i}$. Let us assume for the sake of a contradiction that for some finite sequence $S$ of formulas,

$$
\text { " } S \text { is realized between } x_{i} \text { and } c " \in q_{1}
$$

but

$$
\text { " } S \text { is not realized between } x_{i} \text { and } c \text { " } \in q_{2} \text {. }
$$

By the coheir property of $q_{2}$ there is $a \in A$ such that

$$
C \models \text { " } S \text { is not realized between } a \text { and } c \text { ". }
$$

But $a$ has to be less than every element in $C_{i}$ and thus we get the contradiction " $S$ is not realized between $x_{i}$ and $c " \in q_{1}$. 
2. Prerequisites. We quickly review the crucial definitions and the main results of [15] that we shall use in $\S 3$. Similar results also appeared in [5].

We remark that the set of convex subgroups of an ordered abelian group $G$ is totally ordered by set inclusion; by convention, we consider the empty set as a convex subgroup of $G$.

We consider an element $g$ of an ordered abelian group $G . F_{n}(g)$, the $n$-fundament of $g$, is the largest convex subgroup $C$ of $G$ with $C \cap(g+n G)=\emptyset$. For $n=p^{r}$ we write $F_{p, r}$ instead of $F_{n}$.

An ordered abelian group $G$ is called $n$-regular if any closed interval $[a, b]$ of $G$ which contains at least $n$ elements contains a representative of every congruence class modulo $n G$.

$A_{n}(g)$, the $n$-regular jump of $g$, is the least convex subgroup $C$ of $G$, such that $B(g) / C$ is $n$-regular, where $B(g)$ is the convex subgroup of $G$ generated by $g$.

We recall some basic properties of the groups introduced.

LEMMA 2.1. (1) $A_{n}(g)=A_{n}(m g)$ for all integers $m \neq 0$.

(2) $A_{n}(g+h) \subseteq A_{n}(g) \cup A_{n}(h)$.

(3) If $A_{n}(g) \subset A_{n}(h)$, then $A_{n}(g+h)=A_{n}(h)$.

(4) $F_{n}(g+n h)=F_{n}(g)$.

(5) $F_{n}(g+h) \subseteq F_{n}(g) \cup F_{n}(h)$.

(6) If $F_{n}(g) \subset F_{n}(h)$, then $F_{n}(g+h)=F_{n}(h)$.

Let LOG be the (first-order) language of ordered groups containing the nonlogical symbols: $+,-, 0,<$.

The language of spines, LSP, is the (first-order) language containing as nonlogical symbols, "<" and the unary relation symbols:

$$
A, F, D k, \alpha(p, k, m) \quad \text { for all primes } p, k \geq 1, m \geq 0 .
$$

DEFINITION. For $n \geq 2$ we associate with every ordered abelian group $G$ its $n$-spine $\operatorname{Sp}_{n}(G) . \operatorname{Sp}_{n}(G)$ is the LSP-structure with:

$$
\begin{array}{ll}
\multicolumn{1}{c}{\text { universe }=\left\{A_{n}(g): g \in G\right\} \cup\left\{F_{n}(g): g \in G\right\},} \\
C_{1}<C_{2} & \text { iff } C_{1} \subset C_{2}, \\
A(C) & \text { iff } C=A_{n}(g) \text { for some } g \in G, \\
F(C) & \text { iff } C=F_{n}(g) \text { for some } g \in G, \\
D k(C) & \text { iff } G / C \text { is discrete, } \\
\alpha(p, k, m)(C) & \text { iff } \alpha_{p, k}(\Gamma(n, C)) \geq m .
\end{array}
$$

Here $\Gamma(n, C)$ is the quotient group formed by the subgroup $\Gamma_{2}(n, C)$ of all elements $h \in G$ with $F_{n}(h) \subseteq C$ modulo the subgroup $\Gamma_{1}(n, C)$ of all elements $h \in H$ with $F_{n}(h) \subset C$ and $\alpha_{p, k}(H)$ is the Szmielev invariant given by $\operatorname{dim}\left(p^{k-1} H[p] / p^{k} H[p]\right)$.

The $n$-spines $\operatorname{Sp}_{n}(G)$ are definable over $G$ in the sense made precise in the following lemma.

LEMMA 2.2. For every $n \geq 2, k \geq 0$ and every sequence of LOG-terms $t_{1}(\vec{x}), \ldots, t_{k}(\vec{x})$, every LSP-formula $\chi\left(y_{1}, \ldots, y_{k}, z_{1}, \ldots, z_{k}\right)$ can be translated into a LOG-formula $\varphi(\vec{x})$, such that for all ordered abelian groups $G$ and all $\vec{g} \in G$, $\operatorname{Sp}_{n}(G) \models \chi\left(A_{n}\left(t_{1}(\vec{g})\right), \ldots, A_{n}\left(t_{k}(\vec{g})\right), F_{n}\left(t_{1}(\vec{g})\right), \ldots, F_{n}\left(t_{k}(\vec{g})\right)\right)$ iff $G=\varphi(\vec{g})$. 
The language $\mathrm{LOG}^{*}$ is the definitional extension of LOG obtained by adding the predicates

$$
\begin{gathered}
D(p, r, i)(x), \quad E(n, k)(x), \\
x=1\left(\bmod A_{n}(x)\right), \quad x<1\left(\bmod A_{n}(x)\right), \quad x>1\left(\bmod A_{n}(x)\right)
\end{gathered}
$$

for all $n \geq 2$, all primes $p, r \geq 1,0<i<r$ and $0<k<n$ defined by

$$
\begin{aligned}
D(p, r, i)(x) \leftrightarrow & \exists y\left(p^{r} y=x\right) \vee \exists y\left(F_{p, r}\left(x-p^{i} y\right) \subset F_{p, r}(x)=F_{p, r}(y)\right), \\
E(n, k)(x) \leftrightarrow & \exists y\left(\left(F_{n}(x)=A_{n}(y) \& \text { " } G / A_{n}(y)\right.\right. \text { is discrete and for the } \\
& \text { smallest positive element } z+A_{n}(y) \text { in } G / A_{n}(y) \text { we have } \\
& \left.F_{n}(x-k z) \subset A_{n}(y) "\right) .
\end{aligned}
$$

For $R$ one of the symbols $=,<,>$ :

$x R l\left(\bmod A_{n}(x)\right) \leftrightarrow " G / A_{n}(x)$ is discrete and for its smallest positive element $e+A_{n}(x):\left(x+A_{n}(x)\right) R\left(l \cdot e+A_{n}(x)\right)$ ".

The following properties of these predicates are easily checked:

LEMMA 2.3. (1) If $F_{p, r}(g) \supset F_{p, r}(h)$, then $D(p, r, i)(g+h) \leftrightarrow D(p, r, i)(g)$.

(2) If $F_{p, r}(g)=F_{p, r}(h)$ and $D(p, r, i)(g)$, then $D(p, r, i)(h) \leftrightarrow D(p, r, i)(g-h)$.

(3) If $A_{n}(g) \subset A_{n}(h)$, then $(g+h)=k\left(\bmod A_{n}(g+h)\right) \leftrightarrow h=k\left(\bmod A_{n}(h)\right)$.

(4) $g=k\left(\bmod A_{n}(g)\right) \leftrightarrow m g=m k\left(\bmod A_{n}(m g)\right)$ for $m \neq 0$.

(5) If $F_{n}(g) \subset F_{n}(h)$, then $E(n, k)(g+h) \leftrightarrow E(n, k)(h)$.

(6) $E(n, k)(g) \leftrightarrow E(n, k)(m g)$ for $m \neq 0$.

We can now state the main result from $[\mathbf{1 5}]$.

THEOREM 2.4. For every LOG-formula $\varphi(\vec{x})$ we find:

(i) a natural number $n \geq 2$,

(ii) a LSP-formula $\psi_{0}\left(y_{1}, \ldots, y_{m}, z_{1}, \ldots, z_{r}\right)$,

(iii) a quantifier-free $L O G^{*}$-formula $\psi_{1}(\vec{x})$,

(iv) $L O G$-terms $t_{1}(\vec{x}), \ldots, t_{m}(\vec{x}), s_{1}(\vec{x}), \ldots, s_{r}(\vec{x})$,

such that for all ordered abelian groups $G$ and all $g \in G, G \models \varphi(\vec{g})$ iff $G \models \psi_{1}(\vec{g})$ and

$\mathrm{Sp}_{n}(G) \models \psi_{0}\left(C_{1}, \ldots, C_{m}, D_{1}, \ldots, D_{r}\right) \quad$ for $C_{i}=A_{n}\left(t_{i}(\vec{g})\right), D_{i}=F_{n}\left(s_{i}(\vec{g})\right)$.

PROOF. [15, Theorem 3.6].

3. The number of coheirs in ordered abelian groups. The objective of this section is to prove

THEOREM 3.1. Let $G, H$ be ordered abelian groups, $G \preceq H$, and $p \in S(G)$. Then $p$ has at most $2^{\omega}$ many coheirs in $S(H)$.

Since for every elementary extension $H^{\prime}$ of $H$ every coheir $q \in S(H)$ of $p$ can be extended to a coheir $q^{\prime} \in S\left(H^{\prime}\right), q \subseteq q^{\prime}$, of $p$ (see [10, Corollary 3.8]) we may assume w.l.o.g. that $H$ is $(\operatorname{card} G)^{+}$-saturated.

The proof of Theorem 3.1 will proceed along the following lines.

With each coheir $q \in S(H)$ of $p$ we associate:

* a type $q(0)(x)$ over $H$ consisting of quantifier-free LOG $^{*}$-formulas, 
* for every $n \geq 2$ a type $q(n)\left(y, z_{1}, \ldots, z_{n}\right)$ over $\operatorname{Sp}_{n}(H)$ such that:

3.1 (A) for $q_{1}, q_{2} \in S(H)$ coheirs of $p$ with $q_{1}(0)=q_{2}(0)$ and $q(n)=q(n)$ for all $n \geq 2$ we have already $q_{1}=q_{2}$,

3.1(B) for each $n \geq 2$ there are at most $2^{\omega}$ possibilities for $q(n)$ when $q$ runs through all coheirs of $p$,

$3.1(\mathrm{C})$ there are most $2^{\omega}$ many choices for $q(0)$ when $q \in S(H)$ is to be a coheir of $p$.

Finally the claim of Theorem 3.1 will follow once $3.1(\mathrm{~A}),(\mathrm{B}),(\mathrm{C})$ are proved. The simplest bit is the definition of $q(0)$ :

a quantifier-free $\mathrm{LOG}^{*}$-formula $\varphi(x)$ with parameters from $H$ is in $q(0)$ iff its translation back into an LOG-formula lies in $q$.

For the remaining definitions we keep $n \geq 2$ fixed. The definition of

$$
q(n)\left(y, z_{1}, \ldots, z_{n}\right)
$$

will be effected by defining LOG-terms $\tau_{n}(x), \sigma_{1, n}(x), \ldots, \sigma_{n, n}(x)$ with parameters from $H$ and then

a LSP-formula $\chi\left(y, z_{1}, \ldots, z_{n}\right)$ with parameters from $\operatorname{Sp}_{n}(H)$ is in $q(n)$ iff the formula $\chi\left(A_{n}\left(\tau_{n}(x)\right), F_{n}\left(\sigma_{1, n}(x)\right), \ldots, F\left(\sigma_{n, n}(x)\right)\right)$ belongs to $q$.

Here we have, as we will in the following, tacitly used Lemma 2.2 to ensure that $\chi\left(A_{n}\left(\tau_{n}(x)\right), F_{n}\left(\sigma_{1, n}(x)\right), \ldots, F_{n}\left(\sigma_{n, n}(x)\right)\right)$ is in fact a LOG-statement. We want to stress the important fact that the terms $\tau_{n}, \sigma_{1, n}, \ldots, \sigma_{n, n}$ will depend on $p$ but not on any particular coheir $q$ of $p$.

We first concentrate on the definition of $\tau_{n}(x)$. We call $p(x)$ an extension type with respect to $A_{n}$ if there are $k>0$ and $g \in G$ such that for all $g^{\prime} \in G$

$$
\text { “ } A_{n}(k x+g) \neq A_{n}\left(g^{\prime}\right) " \in p(x),
$$

$k x+g$ is called an extension term of $p(x)$ with respect to $A_{n}$.

LEMMA 3.2. Let $p(x)$ be an extension type with respect to $A_{n}$ with extension term $k x+g$.

(a) Then for all $m \in \mathbf{Z}, m \neq 0, g^{\prime} \in G$,

$$
\text { “ } A_{n}\left(m x+g^{\prime}\right) \supseteq A_{n}(k x+g) " \in p(x) .
$$

(b) Every coheir $q$ of $p$ is again an extension type with respect to $A_{n}$ and we can use the same extension term.

ProOF. (a) " $A_{n}\left(m x+g^{\prime}\right) \subset A_{n}(k x+g)$ " $\in p(x)$ would imply

$$
\text { " } A_{n}(k x+g)=A_{n}\left([m k x+m g]-\left[k m x+k g^{\prime}\right]\right)=A_{n}\left(m g-k g^{\prime}\right) " \in p(x) .
$$

Since $\left(m g-k g^{\prime}\right)$ is an element of $G$ this contradicts $k x+g$ being an extension term of $p(x)$.

(b) Assume to the contrary that for some $h \in H$ " $A_{n}(k x+h)=A_{n}(h)$ " $\in q(x)$. By the coheir property of $q$ there is $g^{*} \in G$ such that $H \models$ " $A_{n}\left(k g^{*}+g\right)=A_{n}(h)$ ". This yields the contradiction " $A_{n}(k x+g)=A_{n}\left(k g^{*}+g\right)$ " $\in p(x)$.

A coheir of a nonextension type may remain so or turn into an extension type. To investigate the situation more closely we introduce the following terminology. 
A nonextension type $p$ is called bounded with respect to $A_{n}$ if there is some $g \in G$ such that for all $g^{\prime} \in G$

$$
\text { “ } A_{n}\left(x+g^{\prime}\right) \supseteq A_{n}(x+g) " \in p(x)
$$

and $g$ is called a bounding element for $p$ with respect to $A_{n}$.

LEMMA 3.3. Let $p(x)$ be a bounded type with respect to $A_{n}$ with bounding element $g$, then:

(i) for all $k \in \mathbf{Z}, k \neq 0, g^{\prime} \in G$,

$$
\text { “ } A_{n}\left(k x+g^{\prime}\right) \supseteq A_{n}(x+g) " \in p(x),
$$

(ii) every coheir $q$ of $p$ is again bounded and we can use the same bounding element.

ProOF. Since $p$ is in particular a nonextension type there is some $a \in G$ such that " $A_{n}(x+g)=A_{n}(a) " \in p(x)$.

(i) Assume for the sake of a contradiction that for some $k \in \mathbf{Z}, k \neq 0$ and $g^{\prime} \in G$ we have " $A_{n}\left(k x+g^{\prime}\right) \subset A_{n}(x+g)$ " $\in p(x)$. Since $p(x)$ is a consistent type over $G$ there is some $g^{*} \in G$ such that $G \models " A_{n}\left(k g^{*}+g^{\prime}\right) \subset A_{n}(a)$ ". But this implies

$$
\text { " } A_{n}\left(x-g^{*}\right)=A_{n}\left(k x-k g^{*}\right)=A_{n}\left(\left[k x+g^{\prime}\right]-\left[k g^{*}+g^{\prime}\right]\right) \subset A_{n}(a)=A_{n}(x+g) " \in p(x)
$$

contradicting $g$ being a bounding element of $p(x)$.

(ii) If we had " $A_{n}(x+h) \subset A_{n}(a)$ " $\in q(x)$ for some $h \in H$ then we could find by the coheir property of $q$ some $g^{*} \in G$ such that $H \models$ " $A_{n}\left(g^{*}+h\right) \subset A_{n}(a)$ ". But this implies the contradiction " $A_{n}\left(x-g^{*}\right)=A_{n}\left([x+h]-\left[g^{*}+h\right]\right) \subset A_{n}(a)$ " $\in p(x)$.

For an unbounded nonextension type $p(x)$ we find a sequence $\left(g_{\alpha}\right)_{\alpha<\lambda}$ of elements from $G$ of length $\lambda \leq \operatorname{card}(G)$ such that for all $\alpha<\beta$

$$
\text { " } A_{n}\left(x+g_{\beta}\right) \subset A_{n}\left(x+g_{\alpha}\right) " \in p(x)
$$

and for all $g \in G$ there is some $\alpha<\lambda$ such that

$$
\text { " } A_{n}\left(x+g_{\alpha}\right) \subset A_{n}(x+g) " \in p(x) .
$$

It is easily seen that for all $\beta<\alpha$

$$
\text { " } A_{n}\left(g_{\beta}-g_{\alpha}\right)=A_{n}\left(g_{\alpha+1}-g_{\alpha}\right) " \in p(x) .
$$

This is the reason for calling $p(x)$ a pseudo-limit type and $\left(g_{\alpha}\right)_{\alpha<\lambda}$ a pseudo-Cauchy sequence for $p$. An element $h$ of some elementary extension $H$ of $G$ is said to be a pseudo-limit of $\left(g_{\alpha}\right)_{\alpha<\lambda}$ if for all $\alpha<\lambda$

$$
H \models \text { " } A_{n}\left(h-g_{\alpha}\right) \subseteq A_{n}\left(g_{\alpha+1}-g_{\alpha}\right) " .
$$

LEMMA 3.4. Let $p(x)$ be a pseudo-limit type with pseudo Cauchy-sequence $\left(g_{\alpha}\right)_{\alpha<\lambda}$ and $h \in H$ a pseudo-limit of $\left(g_{\alpha}\right)_{\alpha<\lambda}$. Then every coheir $q \in S(H)$ of $p$ is an extension type with extension term $x+h$.

ProOF. First we notice that for $g, g^{\prime} \in G$ with " $A_{n}(x+g)=A_{n}\left(g^{\prime}\right)$ " $\in p(x)$ we have " $A_{n}(x+h)=A_{n}([x+g]-[g-h]) \subseteq A_{n}(g)$ " $\in q(x)$, since by choice of $h$ we have $H \vDash " A_{n}(g-h) \subseteq A_{n}\left(g^{\prime}\right)$ ".

Now assume for the sake of a contradiction that we have " $A_{n}(x+h)=A_{n}\left(h^{\prime}\right)$ " $\in$ $q(x)$ for some $h^{\prime} \in H$. By the coheir property of $q$ there is some $g^{*} \in G$ with 
$H \models$ " $A_{n}\left(g^{*}+h\right)=A_{n}\left(h^{\prime}\right)$ ". Since we are dealing with a nonextension type $p$ we can choose $g^{\prime} \in G$ such that " $A_{n}(x-g)=A_{n}\left(g^{\prime}\right)$ " $\in p$. Then

$$
\text { " } A_{n}\left(g^{\prime}\right)=A_{n}\left(x-g^{*}\right)=A_{n}\left([x+h]-\left[g^{*}+h\right]\right) \subseteq A_{n}\left(h^{\prime}\right) " \in q .
$$

DEFINITION OF $\tau_{n}$. (i) If $p(x)$ is an extension type then $\tau_{n}(x)$ is some fixed choice for its extension term.

(ii) If $p(x)$ is a bounded type, then $\tau_{n}(x)=x+b$ with some fixed choice of a bounding element $b$ for $p$.

(iii) If $p(x)$ is a pseudo-limit type with pseudo-Cauchy-sequence $\left(g_{\alpha}\right)_{\alpha<\lambda}$ then we choose some pseudo-limit $h \in H$ of $\left(g_{\alpha}\right)_{\alpha<\lambda}$ and set $\tau_{n}(x)=x+h$.

We now come to consider $n$-fundaments. Since property 2.1(1) has no analogue for $n$-fundaments we have to consider $n$ terms $\sigma_{1, n}, \ldots, \sigma_{n, n}$ instead of one. Each $\sigma_{i, n}$ will be of the form $i x+h$ for some $h \in H$. The details are almost word by word the same as in the definition of $\tau_{n}$; so we allow ourselves to be rather short. In addition to $n \geq 2$, we also keep in the following some $i, 1 \leq i \leq n$, fixed.

DEFINITIONS. (i) $p(x)$ is an extension type with respect to $F_{n}$ and $i$, if there is some $g \in G$ such that for all $g^{\prime} \in G$

$$
\text { “F } F_{n}(i x+g) \neq F_{n}\left(g^{\prime}\right) " \in p(x) .
$$

$g$ is called an extension element for $p$. We set $\sigma_{i, n}=i x+g$ for some fixed choice of an extension element $g$ for $p$.

(ii) $p(x)$ is called a bounded type with respect to $F_{n}$ and $i$, if it is not an extension type and if there is some $g \in G$ such that for all $g^{\prime} \in G$

$$
\text { “ } F_{n}\left(i x+g^{\prime}\right) \supseteq F_{n}(i x+g) " \in p .
$$

$g$ is called a bounding element for $p$. We set $\sigma_{i, n}=i x+g$ for some fixed choice of a bounding element $g$ for $p$.

(iii) $p(x)$ is called a pseudo-limit type with respect to $F_{n}$ and $i$, if it is an unbounded nonextension type. In this case we find a pseudo-Cauchy-sequence $\left(g_{\alpha}\right)_{\alpha<\lambda}$ for $p$, i.e. a sequence $\left(g_{\alpha}\right)_{\alpha<\lambda}$ of elements from $G$ such that for all $g \in G$ there is some $\alpha<\lambda$ with

$$
\text { " } F_{n}(i x+g) \subset F_{n}(i x+g) " \in p
$$

and for all $\alpha<\beta$

$$
\text { " } F_{n}\left(g_{\beta}-g_{\alpha}\right)=F_{n}\left(g_{\alpha+1}-g_{\alpha}\right) " \in p .
$$

We set $\sigma_{i, n}(x)=i x+h$ for some fixed choice $h \in H$ of a pseudo-limit for $\left(g_{\alpha}\right)_{\alpha<\lambda}$.

LEMMA 3.5. (i) Any coheir of an extension type with respect to $F_{n}$ and $i$ is again an extension type and we may use the same extension element.

(ii) Any coheir of a bounded type with respect to $F_{n}$ and $i$ is again a bounded type and we may use the same bounding element.

(iii) Any coheir $q$ of a pseudo-limit type $p$ with respect to $F_{n}$ and $i$ is an extension type and we may use any pseudo-limit of any pseudo-Cauchy-sequence for $p$ as an extension element for $q$.

(iv) In any case we have for all coheirs $q \in S(H)$ of $p$ and any $h \in H$,

$$
\text { " } F_{n}(i x+h) \supseteq F_{n}\left(\sigma_{i, n}(x)\right) " \in q .
$$

PROOFS. Analogous to the proofs of Lemmas 3.2-3.4. 
ProOF OF 3.1(A). Let $H^{*}$ be an elementary extension of $H, h_{1}^{*}, h_{2}^{*} \in H^{*}$ realizing $q_{1}, q_{2}$ respectively and consider a LOG-formula $\varphi(x)$ with parameters from $H$. We have to show

$$
H \vDash \varphi\left(h_{1}^{*}\right) \leftrightarrow \varphi\left(h_{2}^{*}\right) .
$$

By Theorem 2.4 we can equivalently replace $\varphi$ by a quanitifer-free $\mathrm{LOG}^{*}$-formula $\varphi_{0}(x)$ with parameters from $H$ and a LSP-formula

$$
\varphi_{1}\left(A_{n}\left(t_{1}(x)\right), \ldots, A_{n}\left(t_{m}(x)\right), F_{n}\left(s_{1}(x)\right), \ldots, F_{n}\left(s_{r}(x)\right)\right)
$$

with parameters from $\operatorname{Sp}_{n}(H)$ for an appropriate $n \geq 2$. Now $H \models \varphi_{0}\left(h_{1}^{*}\right) \leftrightarrow$ $\varphi_{0}\left(h_{2}^{*}\right)$ follows immediately from $q_{1}(0)=q_{2}(0)$. The corresponding equivalence for $\varphi_{1}$ will follow from $q_{1}(n)=q_{2}(n)$ when we succeed to verify the following:

(1) For all $j, 1 \leq j \leq m$, either

$$
A_{n}\left(t_{j}\left(h_{1}^{*}\right)\right)=A_{n}\left(t_{j}\left(h_{2}^{*}\right)\right) \in \operatorname{Sp}_{n}(H)
$$

or

$$
A_{n}\left(t_{j}\left(h_{1}^{*}\right)\right)=A_{n}\left(\tau_{n}\left(h_{1}^{*}\right)\right) \text { and } A_{n}\left(t_{j}\left(h_{2}^{*}\right)\right)=A_{n}\left(\tau_{n}\left(h_{2}^{*}\right)\right) .
$$

(2) For all $j, 1 \leq j \leq r$, either

$$
F_{n}\left(s_{j}\left(h_{1}^{*}\right)\right)=F_{n}\left(s_{j}\left(h_{2}^{*}\right)\right) \in \operatorname{Sp}_{n}(H)
$$

or for some $i, 1 \leq i \leq n$,

$$
F_{n}\left(s_{j}\left(h_{1}^{*}\right)\right)=F_{n}\left(\sigma_{i, n}\left(h_{1}^{*}\right)\right) \quad \text { and } \quad F_{n}\left(s_{j}\left(h_{2}^{*}\right)\right)=F_{n}\left(\sigma_{i, n}\left(h_{2}^{*}\right)\right) .
$$

Verification of (1). We fix $j$ and use the notation $t_{j}=k x+h, \tau_{n}=m x+a$. It follows from Lemmas 3.2-3.4 that

$$
\text { “ } A_{n}(k x+h) \supseteq A_{n}\left(\tau_{n}(x)\right) " \in q_{1} \cap q_{2} .
$$

A simple computation using Lemma 2.1(3) shows for $i=1,2$

$$
\text { “ } A_{n}(k x+h) \supset A_{n}(m x+a) " \in q_{i}(x) \quad \text { iff } \quad \text { " } A_{n}(m h-k a) \supset A_{n}(m x+a) " \in q_{i}(x) \text {. }
$$

Since the assumption $q_{1}(n)=q_{2}(n)$ implies

$$
\text { " } A_{n}(m h-k a) \supset A_{n}(m x+a) " \in q_{1}(x) \quad \text { iff } \quad \text { " } A_{n}(m h-k a) \supset A_{n}(m x+a) " \in q_{2}(x)
$$

we see that either

" $A_{n}(k x+h)=A_{n}(m h-k a) " \in q_{1} \cap q_{2} \quad$ or $\quad$ " $A_{n}(k x+h)=A_{n}\left(\tau_{n}(x)\right) " \in q_{1} \cap q_{2}$.

The verification of (2) proceeds analogously.

PROOF OF 3.1(B). To begin with let us remark that from the assumption $G \preceq H$ it follows using Lemma 2.2 that the maps $f_{n}: \operatorname{Sp}_{n}(G) \rightarrow \operatorname{Sp}_{n}(H)$ defined by

$$
f_{n}\left(A_{n}^{G}(g)\right)=A_{g}^{H}(g), \quad f_{n}\left(F_{n}^{G}(g)\right)=F_{n}^{H}(g)
$$

are well defined elementary embeddings. We will identify $\operatorname{Sp}_{n}(G)$ with its isomorphic image under $f_{n}$; thus look upon $\operatorname{Sp}_{n}(G)$ as an elementary substructure of $\mathrm{Sp}_{n}(H)$.

By Fact $\mathrm{A}$ of $\S 1$ it suffices to prove for each $n \geq 2$ that for $y$ and each $z_{i}, 1 \leq$ $i \leq n$, there are at most 2 possibilities for the cut determined by $q(n)$ and $y$ (respectively by $q(n)$ and $z_{i}$ ) as $q$ runs through all coheirs of $p$. We shall verify this for the variable $y$; the verification for the variables $z_{i}$ are entirely analogous. 
If $p$ is not a pseudo-limit type then the parameter in $\tau_{n}(x)$ belongs to $G$ and we may define the type in $S_{1}\left(\operatorname{Sp}_{n}(G)\right)$ :

$$
\begin{array}{r}
p_{1, n}(y)=\left\{\chi(y): \chi(y) \text { a LSP-formula with parameters from } \operatorname{Sp}_{n}(G)\right. \\
\text { such that } \left.\chi\left(A_{n}\left(\tau_{n}(x)\right)\right) \text { belongs to } p\right\} .
\end{array}
$$

For every coheir $q$ of $p$ in $\operatorname{Sp}_{n}(H)$ we get by Lemma 2.2 that $q_{1, n}(y)$ is a coheir of $p_{1, n}$, where $q_{1, n}(y)$ is the restriction of $q(n)$ to formulas containing at most the free variable $y$.

If $p$ is a pseudo-limit type with pseudo-Cauchy-sequence $\left(g_{\alpha}\right)_{\alpha<\lambda}, \lambda \leq \operatorname{card} G$, then $\tau_{n}(x)=x+h$ for some $h \in H, h$ a pseudo-limit of $\left(g_{\alpha}\right)_{\alpha<\lambda}$. Let $C_{0}=\{C \in$ $\operatorname{Sp}_{n}(G):\left(" C<A_{n}(x+g)\right.$ " $\left.\in p\right\}$ and $C_{1}=\operatorname{Sp}_{n}(G) \backslash C_{0}$. For any coheir $q \in S(H)$ of $p$ we must have for all $C_{0} \in C_{0}, C_{1} \in C_{1}$

$$
\text { " } C_{0}<A_{n}(x+h)<C_{1} " \in q(x) .
$$

" $A_{n}(x+h)<C_{1}$ " $\in q(x)$ is a simple consequence of the fact that $h$ is a pseudo-limit of $\left(g_{\alpha}\right)_{\alpha<\lambda}$. Assume that " $A_{n}(x+h) \leq C_{0}$ " $\in q(x)$. By the coheir property we have for some $g^{\prime} \in G H \models$ " $A_{n}\left(g^{\prime}+h\right) \leq C_{0}$ ". Since $p$ is unbounded with respect to $A_{n}$ we have for some $\alpha<\lambda$ : " $A_{n}(x+g) \subset A_{n}\left(g+g^{\prime}\right)$ " $\in p(x)$. Thus

$$
\text { " } A_{n}(g-h)=A_{n}\left(g+g^{\prime}-\left(g^{\prime}+h\right)\right)=A_{n}\left(g+g^{\prime}\right) " \in q(x) .
$$

But this would imply inconsistency of $q(x)$ since $C_{0}<A_{n}\left(g+g^{\prime}\right)=A_{n}(g-h) \leq C_{0}$.

Next we claim that for any coheir $q \in S(H)$ of $p$ either " $C<A_{n}(x+h)$ " $\in q$ for all $C \in \operatorname{Sp}_{n}(H)$ with $C_{0}<C<C_{1}$ or " $A_{n}(x+h)<C$ " $\in q$ for all $C \in \operatorname{Sp}_{n}(H)$ with $C_{0}<C<C_{1}$. Assume to the contrary that for $C, C^{\prime} \in \operatorname{Sp}_{n}(H)$ with $C_{0}<$ $C<C^{\prime}<C_{1}$ we have "C<An $(x+h)<C^{\prime \prime} \in q$. By the coheir property there exists some $g \in G$ such that $H \models C<A_{n}(g+h)<C^{\prime}$. For some $\alpha<\lambda$ we have " $A_{n}\left(x+g_{\alpha}\right)<A_{n}(x-g)=A_{n}\left(g+g_{\alpha}\right)$ " $\in p$. For the pseudo-limit $h$ we have $A_{n}\left(h-g_{\alpha}\right) \leq A_{n}\left(g_{\alpha+1}-g_{\alpha}\right)$. Thus $A_{n}(h+g)=A_{n}\left(h-g_{\alpha}+g+g_{\alpha}\right)=A_{n}\left(g+g_{\alpha}\right)$. This is a contradiction since there are no elements of $\operatorname{Sp}_{n}(G)$ between $C$ and $C^{\prime}$.

PROOF OF 3.1(C). We introduce the abbreviations:

$\Sigma_{1}=$ the set of inequalities $k x \leq h, h \geq k x$ with $k \in \mathbf{Z}, k \neq 0$ and $h \in H$.

$\Sigma_{2}=$ the set of formulas

$$
(l x+h)=k\left(\bmod A_{n}(l x+h)\right), \neg(l x+h)=k\left(\bmod A_{n}(l x+h)\right) \text { for } h \in H
$$

and all relevant $n, k, l$.

$\Sigma_{3}=$ the set of formulas $D(p, r, i)(l x+h), \neg D(p, r, i)(l x+h)$ for $h \in H$ and all relevant $p, r, i, l$.

$\Sigma_{4}=$ the set of formulas $E(p, r, k)(l x+h), \neg E(p, r, k)(l x+h)$ for $h \in H$ and all relevant $p, r, k, l$.

Since every quantifier-free $\mathrm{LOG}^{*}$-formula is a Boolean combination of formulas from $\Sigma_{1} \cup \cdots \cup \Sigma_{4}$ it suffices to show for $i, 1 \leq i \leq 4$,

$$
\operatorname{card}\left\{q(0) \cap \Sigma_{i}: q \in S(H) \text { a coheir of } p\right\} \leq 2^{\omega} .
$$

Part 1. $q \cap \Sigma_{1}$ is completely determined by the $\omega$ many cuts

$$
C_{k, u}=\{h \in H:(k x<h) \in q\}, \quad C_{k, l}=\{h \in H:(k x>h) \in q\} .
$$

By Theorem 1.2 there are for each $k$ at most two possibilities for the cut $\left(C_{k, u}, C_{k, l}\right)$. 
Part 2. We show

(2) for any two coheirs $q_{1}, q_{2} \in S(H)$ of $p$ such that

(A) for all $n \geq 2: q_{1}(n)=q_{2}(n)$ and

(B) for all $n \geq 2$ and all $k \geq 1$ :

$$
\tau_{n}(x)=k\left(\bmod A_{n}\left(\tau_{n}(x)\right)\right) \in q_{1} \quad \text { iff } \quad \tau_{n}(x)=k\left(\bmod A_{n}\left(\tau_{n}(x)\right)\right) \in q_{2}
$$

it follows that $q_{1}(0) \cap \Sigma_{2}=q_{2}(0) \cap \Sigma_{2}$.

Now obviously (2) implies (1) for $i=2$ by $3.1(\mathrm{~B})$. To prove (2) we denote $\tau_{n}(x)$ more detailed by $m x+a$ and notice first that by Lemma 2.3 and the fact that we have " $A_{n}(l x+h) \supseteq A_{n}\left(\tau_{n}(x)\right)$ " $\in q$ in any case, we get the following criterion:

$(l x+h)=k\left(\bmod A_{n}(l x+h)\right) \in q$ iff

(i) " $A_{n}(l x+h) \supset A_{n}(m x+a) \&(m h-l a)=k m\left(\bmod A_{n}(m h-l a)\right)$ " $\in q$ or

(ii) " $A_{n}(l x+h)=A_{n}(m x+a) \&(l x+h)=k\left(\bmod A_{n}(l x+h)\right)$ " $\in q$.

If $(l x+h)=k\left(\bmod A_{n}(l x+h)\right) \in q_{1}$ and (i) applies then assumption (A) implies immediately $(l x+h)=k\left(\bmod A_{n}(l x+h)\right) \in q_{2}$. If (ii) applies and $p(x)$ is an extension or a pseudo-limit type with respect to $A_{n}$ then " $A_{n}(m h-l a) \subset$ $A_{n}(m x+a)$ " $\in q_{1}$ and by (A) also " $A_{n}(m h-l a) \subset A_{n}(m x+a)$ " $\in q_{2}$. But now

$$
\begin{aligned}
(l x+h)=k\left(\bmod A_{n}(\right. & l x+h)) \in q_{1} \\
& \text { iff }(l m x+m h)=k m\left(\bmod A_{n}(l x+h)\right) \in q_{1}, \\
& \text { iff }(l m x+l a)=k m\left(\bmod A_{n}(m x+a)\right) \in q_{1}, \\
& \text { iff } k m=l \cdot l^{\prime} \&(m x+a)=l^{\prime}\left(\bmod A_{n}(m x+a)\right) \in q_{1}, \\
& \text { iff }(m x+a)=l^{\prime}\left(\bmod A_{n}(m x+a)\right) \in q_{2} \quad(\text { by }(\mathrm{B})), \\
& \text { iff }(l x+h)=k\left(\bmod A_{n}(l x+h)\right) \in q_{2} .
\end{aligned}
$$

If $p(x)$ is a bounded type we will argue that (ii) cannot happen. Indeed in this case we have " $A_{n}(m x+a)=A_{n}(g)$ " $\in q_{1} \cap q_{2}$ for some $g \in G$. If " $(l x+h)=$ $k\left(\bmod A_{n}(l x+h)\right) \& A_{n}(m x+a)=A_{n}(g) " \in q$, then we may assume w.l.o.g. that $G \models g=1\left(\bmod A_{n}(g)\right)$. But this yields " $A_{n}(l x+h-k g) \subset A_{n}(m x+a) " \in q$ contradicting Lemma 3.2.

PART 3. We show that

(3) for any two coheirs $q_{1}, q_{2} \in S(H)$ of $p$ such that

(A) for all $n \geq 2, q_{1}(n)=q_{2}(n)$ and

(B) for all $p, r, i$ and $k$

$$
D(p, r, i)\left(\sigma_{k, p^{r}}\right) \in q_{1} \quad \text { iff } \quad D(p, r, i)\left(\sigma_{k, p^{r}}\right) \in q_{2}
$$

we have $q_{1} \cap \Sigma_{3}=q_{2} \cap \Sigma_{3}$.

Obviously (3) implies (1) for $i=3$. We write more detailed $k x+a$ for $\sigma_{k, p^{r}}$. To prove (3) we notice first that by Lemma 3.5(iv) we have the following criterion for all coheirs $q$ of $p$ and all $h \in H$ :

$D(p, r, i)(k x+h) \in q$ iff

(i) " $F_{p, r}(k x+h) \supset F_{p, r}(k x+a) \& D(p, r, i)(h-a)$ " $\in q$ or

(ii) " $F_{p, r}(k x+h)=F_{p, r}(k x+a) \& D(p, r, i)(k x+h)$ " $\in q$.

If $D(p, r, i)(k x+h) \in q_{1}$ and (i) applies then by $(3)(\mathrm{A})$ " $F_{p, r}(k x+h) \supset$ $F_{p, r}(k x+a) " \in q_{2}$ and we obtain immediately $D(p, r, i)(k x+h) \in q_{2}$. If (ii) applies and $p(x)$ is an extension or a pseudo-limit type with respect to $F_{p, r}$ and $k$, then 
" $F_{p, r}(h-a) \subset F_{p, r}(k x+a) " \in q_{1}$, thus by $(3)(\mathrm{A})$ also " $F_{p, r}(h-a) \subset F_{p, r}(k x+a) " \in$ $q_{2}$. But now

$$
\begin{array}{ll}
D(p, r, i)(k x+h) \in q_{1} & \text { iff } D(p, r, i)(k x+a) \in q_{1}, \\
& \text { iff } D(p, r, i)(k x+a) \in q_{2} \quad(\text { by }(\mathrm{B})), \\
& \text { iff } D(p, r, i)(k x+h) \in q_{2} .
\end{array}
$$

If $p(x)$ is a bounded type we have " $F_{p, r}(k \dot{x}+h)=F_{p, r}(g)$ " $\in q_{1}$ for some $g \in H$. By the coheir property of $q_{1}$ there is $g_{0} \in G$ such that

$$
H \models F_{p, r}\left(k g_{0}+h\right)=F_{p, r}(g) \& D(p, r, i)\left(k g_{0}+h\right) .
$$

Thus by Lemma $2.3 D(p, r, i)\left(k x-k g_{0}\right) \in p \subseteq q_{1} \cap q_{2}$ and further $D(p, r, i)(k x+h) \in$ $q_{2}$.

Part 4. Follows along the very same line as Part 2.

\section{REFERENCES}

1. F. Delon, A counterexample concerning the number of coheirs, private communication by B. Poizat.

2. _. Types sur $C((X))$, Théories Stables, $2^{\circ}$ année, IHP, Paris, 1981.

3. J.-L. Duret, Les corps pseudo-finis ont la propriété d'independance, C. R. Acad. Sci. Paris Sér. A 290 (1980), 981-903.

4. S. Feferman and R. L. Vaught, The first-order properties of products of algebraic systems, Fund. Math. 4 (1959), 57-105.

5. Y. Gurevich, The decision problem for some algebraic theories, Doctoral Dissertation, Sverdlovsk, U.S.S.R., 1968.

6. __ Elementary properties of ordered abelian groups, Trans. Amer. Math. Soc. 46 (1965), 165192.

7. __ Expanded theory of ordered abelian groups, Ann. Math. Logic 12 (1977), 193-228.

8. H. J. Keisler, The stability function of a theory, J. Symbolic Logic 43 (1978), 481-486.

9. __ Six classes of theories, J. Austral. Math. Soc. Ser. A 21 (1976), 257-266.

10. D. Lascar and B. Poizat, An introduction to forking, J. Symbolic Logic 44 (1979), 178-198.

11. M. Parigot, Théories d'arbres, Doctoral Dissertation, Paris, 1981.

12. B. Poizat, Théories instables, J. Symbolic Logic 46 (1981), 513-522.

13. M. Rubin, Theories of linear order, Israel J. Math. 17 (1974), 392-443.

14. P. H. Schmitt, Model theory of ordered abelian groups, Habilitationsschrift, Heidelberg, 1982.

15. __ Elementary properties of ordered abelian groups (to appear).

16. S. Shelah, Stability, the f.c.p. and superstability: model theoretic properties of formulas in first-order theory, Ann. Math. Logic 3 (1971), 271-362.

DEPARTMENT OF COMPUTER AND COMMUNICATION SCIENCES, UNIVERSITY OF MiChigan, ANN ARBOR, MiChIGAN 48109

Mathematisches Institut, Universität Heidelberg, 6900 Heidelberg 1, FedERAL REPUBLIC OF GERMANY 